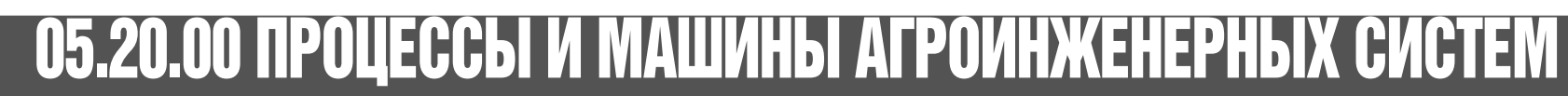

УДК 631.3

DOI 10.18286/1816-45-2017-4-179-183

\section{ОСОБЕННОСТИ ДВИЖЕНИЯ ЗЕРНА В УСТРОЙСТВЕ ДЛЯ ЕГО ВОРОШЕНИЯ}

Исаев Юрий Михайлович, доктор технических наук, профессор, заведующий кафедрой «Математика и физика»

Семашкин Николай Михайлович, кандидат технических наук, доцент кафедры «Математика и физика»

Каленков Сергей Александрович, аспирант кафедры «Математика и физика»

ФГБОУ ВО УльянОвскИй ГАУ

432017, г. Ульяновск, бульвар Новый Венеи, 1; тел.: 8 (84231) 55-95-49;

e-mail:emotion.snm@mail.ru

Ключевые слова: перемещение, ворошение, пропускная способность, уравнения регрессии, скорость перемещения зерна.

В статье приведен анализ теоретических и практических исследований параметров устройства для вертикального перемещения зерна в насыпи. В результате получены оптимальные параметры устройства вертикального перемещения зерна и геометрические характеристики спирали. Зерно, которое заполняет межвитковое пространство спирали, находится в стесненных условиях, вследствие чего исследование движения зернового материала спирально-винтовым рабочим органом представляет одну из сложных экспериментальных и теоретических задач. В теоретической части статьи приведен анализ движения сыпучего потока зерна, для чего рассмотрены выражения пропускной способности устройства ворошения и скоростей движения зерна. В исследованиях на устройство устанавливали спиральный винт со следующими параметрами: внешний радиус спирального винта $r_{2}=35$ мм; шаг винтовой линии спирали $s=0,07$ м; диаметр проволоки, из которой изготавливали спираль, $d_{n}=0,008$ м, полная высота спирали $H=2$ м. Исследования устройства проводили в бурте пшеницы с насыпной плотностью $\rho=780$ кг $/ \mathrm{M}^{3}$. В итоге получили оптимальную скорость перемещения устройства для ворошения $U=0,68 \mathrm{M} / \mathrm{мин,} \mathrm{которая} \mathrm{достигается} \mathrm{при} \mathrm{частоте} \mathrm{вращения}$ спирали $\omega=930$ мин $^{-1}$. При этом пропускная способность устройства $Q=1,1$ кг/с. Сравнение полученных экспериментальных и теоретических данных показало, что сходимость результатов составляет $94 \%$. Результаты экспериментальных исследований доказывают справедливость предложенных теоретических зависимостей.

\section{Введение}

При хранении зерна возникает множество проблем, связанных с его перемещением, большими материальными затратами, отсутствием соответствующих средств механизации и т. д. Одним из основных показателей при хранении зерна является его температура, так как самосогревание зерна внутри насыпи нарушает процесс его хранения. Для предотвращения самосогревания нужно использовать сушку зерна, активное вентилирование и т. п. Одним из лучших способов предотвращения самосогревания зерна и последующей его порчи является перемешивание всего бурта устройством для ворошения зерна с рабочим органом в виде спирального винта.

При этом разработка нового простого и надежного в эксплуатации устройства ворошения зерна на базе спирально-винтового рабочего органа является актуальной задачей $[1,2,3]$. 


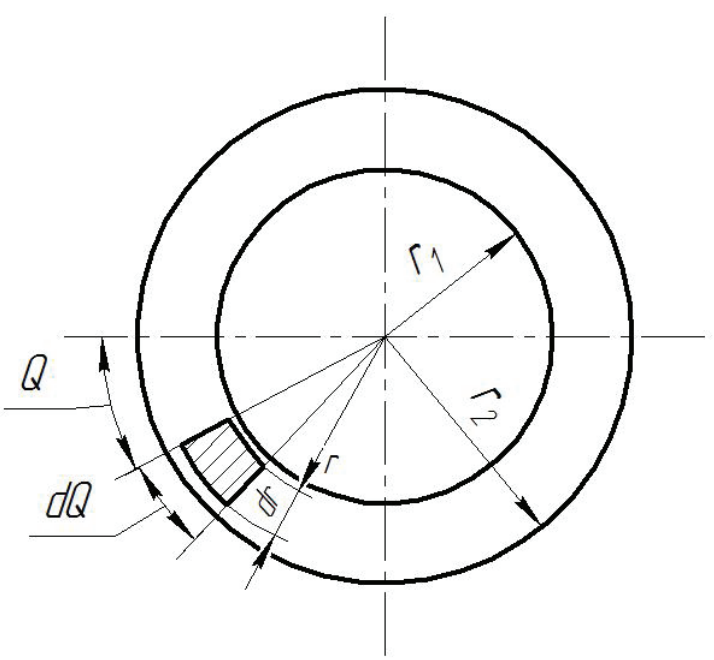

a)

Рис. 1 - К определению средней скорости зерна

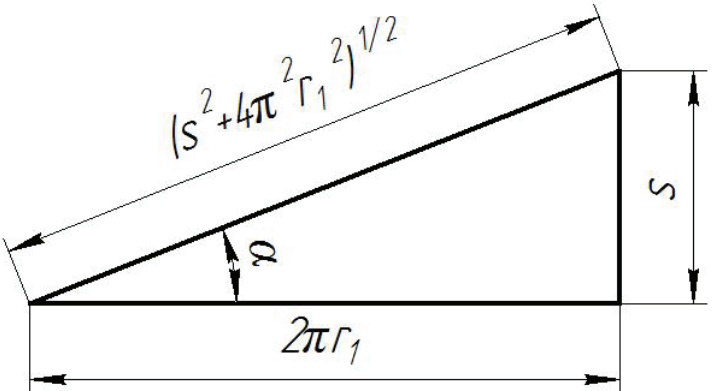

б)

\section{Объекты и методы исследований}

Зерно, которое заполняет межвитковое пространство спирали, находится в стесненных условиях, вследствие чего исследование движения зернового материала спирально-винтовым рабочим органом представляет одну из сложных экспериментальных и теоретических задач [4, 5].

Переходя к движению сыпучего потока зерна, запишем формулу для определения пропускной способности, кг/с, устройства для ворошения сыпучего материала [6]:

$$
Q=k_{n} \cdot F \cdot v_{0 c p} \cdot \rho,
$$

где $k_{n}$ - коэффициент пропускной способности; $F$ - рабочая площадь поперечного сечения спирали, м $^{2} \vartheta_{\text {оср }}$ - средняя осевая скорость материала, м/с; $\rho$ - насыпная плотность зерна, кг/м³.

Под коэффициентом пропускной способности $k_{n}$ следует понимать число, равное или меньшее единицы, показывающее ту часть рабочей площади поперечного сечения спирали $F$, в которой условно перемещается весь материал со средней скоростью $v_{\text {оср }}$

Придадим формуле (1) развернутый вид:

$Q=\pi \cdot v_{0 c p} \cdot \rho \cdot k_{n}\left(r_{2}^{2}-r_{1}^{2}\right)$,

где $r_{2}$ - внешний радиус спирального винта, м; $r_{1}$ - внутренний радиус спирального винта, м.

Выделим на проекции винтовой поверх- ности спирали на плоскость, перпендикулярную оси z, элементарную бесконечно малую площадку $d F=r d r d Q$, где $r-$ расстояние от цен-

тра вращения спирали до элементарной площадки, м (рис. 1).

Считая для элементарной площадки скорости $v_{0} u v_{s}$ постоянными, определим среднюю осевую скорость зернового материала на элементарной площадке $d r[7,8]$ (рис. 1 a):

$$
v_{0 c p}=\frac{\iint_{F} v_{0} d F}{\iint_{F} d F}=\frac{\int_{0}^{2 \pi} d Q \int_{r_{1}}^{r_{2}} v_{0} r d r}{\int_{0}^{2 \pi} d Q \int_{r_{1}}^{r_{2}} r d r},
$$

где $v_{0}$ - осевая скорость каждого единичного зерна, находящегося на площадке $d r$, м/с.

Средняя окружная скорость единичного зерна по площади сечения спирали

$$
v_{s c p}=\frac{\iint_{F} v_{s} d F}{\iint_{F} d F}=\frac{\int_{0}^{2 \pi} d Q \int_{r_{1}}^{r_{2}} v_{s} r d r}{\int_{0}^{2 \pi} d Q \int_{r_{1}}^{r_{2}} r d r},
$$

где $v_{s}$ - переносная скорость каждого единичного зерна, находящегося на площадке $d r$, $\mathrm{M} / \mathrm{C}$.

Заменим в формулах (3) и (4) тригонометрические функции зависимостями, которые можно записать, рассмотрев развертку винтовой линии (рис. 1 б): 


$$
\begin{aligned}
& \sin \alpha=\frac{s}{\sqrt{s^{2}+4 \pi^{2} r^{2}}}, \\
& \cos \alpha=\frac{2 \pi r}{\sqrt{s^{2}+4 \pi^{2} r^{2}}},
\end{aligned}
$$

где $s$ - шаг винта, м.

Для упрощения математических преобразований обозначим $c=s / 2 \pi$ - часть шага винтовой поверхности спирали, приходящуюся на один радиан поворота образующей, и выразим значения $\sin \alpha_{\text {и }} \cos \alpha$ через $c$ :

$$
\sin \alpha=\frac{c}{\sqrt{c^{2}+r^{2}}}, \cos \alpha=\frac{r}{\sqrt{c^{2}+r^{2}}} .
$$

Тогда соответственно осевая и переносная скорости:

$$
\begin{aligned}
& v_{0}=\frac{\omega c r \operatorname{tg} \beta}{c+\operatorname{tg} \beta \cdot r}, \\
& v_{s}=\frac{\omega c r}{c+\operatorname{tg} \beta \cdot r},
\end{aligned}
$$

где $\omega$ - частота вращения спирали, мин ${ }^{-1}$; $\beta$-угол между векторами скоростей $v_{0} u v_{s^{\prime}}$ град. Обозначив $q=\operatorname{tg} \beta$ и подставив значения $v_{0} и v_{s}$ в выражения (5) и (6), получим: $v_{0 c p}=\frac{\omega c}{2 q^{2}\left(r_{2}^{2}-r_{1}^{2}\right)}\left[q^{2}\left(r_{2}^{2}-r_{1}^{2}\right)-2 q c\left(r_{2}-r_{1}\right)+c^{2} \ln \frac{c+q r_{2}}{c+q r_{1}}\right]$.

Средняя окружная скорость зерна:

$$
v_{s c p}=\frac{\omega c}{2 q^{3}\left(r_{2}^{2}-r_{1}^{2}\right)}\left[q^{2}\left(r_{2}^{2}-r_{1}^{2}\right)-2 q c\left(r_{2}-r_{1}\right)+c^{2} \ln \frac{c+q r_{2}}{c+q r_{1}}\right] \text {. }
$$

Формулы (7) и (8) выведены с учетом того, что межвитковое пространство спирали полностью заполнено зерном.

Для спиральных винтов, применяющихся для ворошения зерна, с шагом спирали от $50 \mathrm{~mm}$ до 100 мм и коэффициентом $t=s /\left(2 r_{2}\right)=0,7 \ldots 1,3$ выражение (7) можно значительно упростить.

Значение $\ln \frac{c+q r_{2}}{c+q r_{1}}$ можно разложить в ряд и, пренебрегая величинами малого порядка, записать в следующем виде $[9,10]$ :

$$
\ln \frac{c+q r_{2}}{c+q r_{1}} \approx \frac{q\left(r_{2}-r_{1}\right)}{c+q r_{1}}
$$

Тогда выражения (7) и (8) примут вид:

$$
\begin{gathered}
v_{0 c p}=\frac{\omega c}{2 q^{2}\left(r_{2}^{2}-r_{1}^{2}\right)}\left[q^{2}\left(r_{2}^{2}-r_{1}^{2}\right)-2 q c\left(r_{2}-r_{1}\right)+c^{2} \frac{q\left(r_{2}-r_{1}\right)}{c+q r_{1}}\right] \\
v_{0 c p}=\frac{\omega c}{2}\left[1-\frac{2 c r_{1}}{\left(c+q r_{1}\right)\left(r_{2}+r_{1}\right)}\right] ; \\
v_{s c p}=\frac{\omega c}{2 q}\left[1-\frac{2 c r_{1}}{\left(c+q r_{1}\right)\left(r_{2}+r_{1}\right)}\right] .
\end{gathered}
$$

Подставив значение $U_{0 c p}$ в (2), получим:

$$
Q=\frac{\pi \omega c \rho k_{n}}{2}\left[\left(r_{2}^{2}-r_{1}^{2}\right)-\frac{2 c r_{1}\left(r_{2}-r_{1}\right)}{\left(c+q r_{1}\right)}\right] \text {. }
$$

Пропускная способность является сложной функцией величины шага. Если известны геометрические и кинематические характеристики спирали, насыпная плотность зерна, то, подставляя значения этих параметров в формулу (11), можно определить пропускную способность устройства для ворошения зерна.

\section{Результаты исследований}

В устройство для ворошения зерна устанавливали спиральный винт со следующими параметрами: внешний радиус спирального винта $r_{2}=35$ мм; шаг винтовой линии спирали $s=0,07$ м; диаметр проволоки, из которой изготавливали спираль, $d_{n}=0,008$ м, полная высота спирали $H=2$ м. Устройство исследовали в бурте пшеницы с насыпной плотностью $\rho=780 \mathrm{kr} / \mathrm{m}^{3}$. По результатам исследований построены зависимости скорости перемещения $v$ устройства ворошения по поверхности бурта зерна при определенной пропускной способности $Q$ устройства от частоты вращения $\omega$ спирали (рис. 2).

При этом линейное уравнение регрессии скорости перемещения устройства по поверхности насыпи зерна имеет вид:

$$
v(\omega)=0,85 \cdot 10^{-3} \omega-0,303 \text {. }
$$

На рис. 2 изображены зависимости пропускной способности $Q$ (сплошная линия) и скорости перемещения $v$ устройства (точки) для ворошения зерна от частоты вращения спирали $\omega$.

Также были проведены экспериментальные исследования влияния шага спирали при ее постоянном диаметре на пропускную способность устройства при различных частотах вращения спирали (рис. 3).

По результатам исследований по изучению влияния шага $s$ спирали на пропускную спо- 


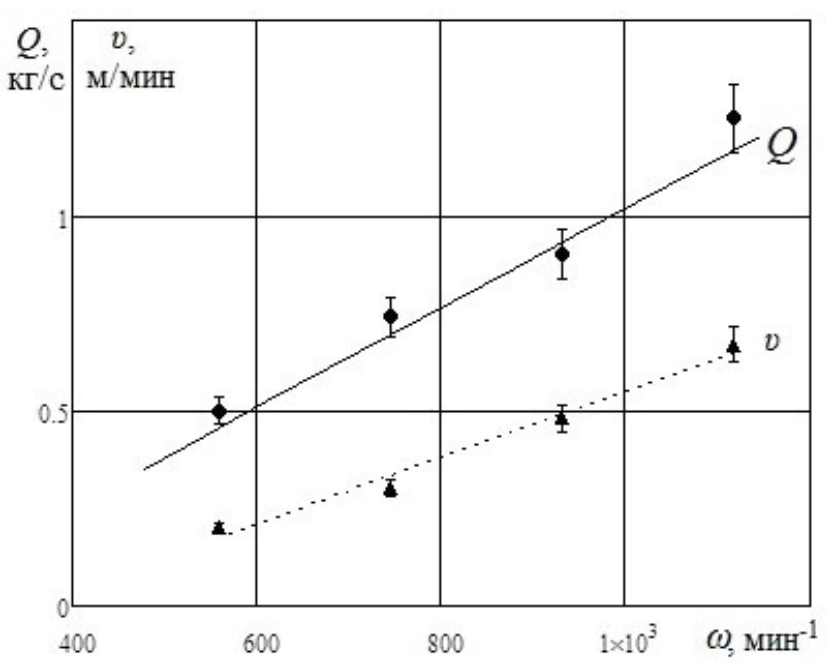

Рис. 2 - Скорость перемещения $v$ устройства для ворошения по поверхности зерна и пропускная способность $Q$ устройства в зависимости от частоты вращения $\omega$ спирали

собность устройства при различных частотах вращения $\omega$ также получено уравнение (13) в натуральных значениях факторов:

$Q=0,312-1,02 \cdot 10^{-3} \omega-5,8 \cdot 10^{-7} \omega^{2}+4,67 s+0,018 s \omega$

Уравнение регрессии (13) в кодированных значениях факторов имеет вид:

$y=0,606+0,235 x_{1}+0,041 x_{1}^{2}+0,218 x_{2}+0,104 x_{1} x_{2}$

где $y$ - пропускная способность устройства, кг/с; $x_{1}$ - частота вращения спирали; $x_{2}-$ шаг спирали.

Уравнения регрессии (13) и (14) позволяют подобрать частоту вращения спирального винта для обеспечения требуемой пропускной способности устройства при различном шаге спирали.

\section{Выводы}

При шаге спирали $s=0,070$ м оптимальная скорость перемещения устройства для ворошения $U=0,68 \mathrm{M} /$ мин достигается при частоте вращения спирали $\omega=930$ мин $^{-1}$. При этом пропускная способность устройства $Q=1,1$ кг/с. Сравнение полученных экспериментальных и теоретических данных показало, что сходимость результатов составляет 94 \%. Следовательно, результаты экспериментальных исследований доказывают справедливость предложенных теоретических зависимостей.

\section{Библиографический список}

1. Курдюмов, В.И. Обеззараживание зер-

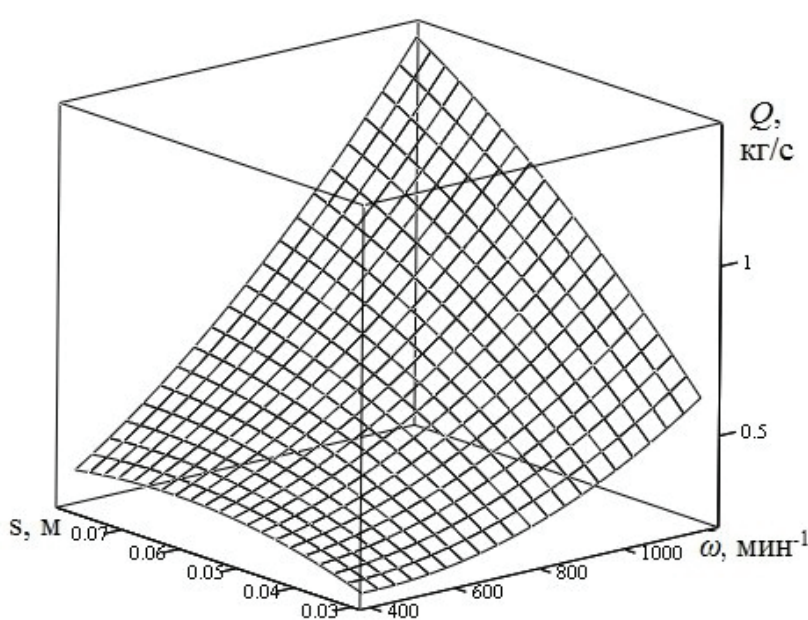

Рис. 3 - Зависимость пропускной способности $Q$ от шага спирали $s$ и частоты её вращения $\omega$

на в установке комбинированного типа / В.И. Курдюмов, А.А. Павлушин, С.А. Сутягин // Аграрная наука как основа продовольственной безопасности региона Материалы 66-й международной научно-практической конференции. - Ульяновск, УГСХА, 2015. - С. 181-183.

2. Курдюмов, В.И. Повышение качества сушки зерна в установке контактного типа / В.И. Курдюмов, А.А. Павлушин, С.А. Сутягин // Инновации в сельском хозяйстве. -2015. - № 3 (13). - С. 79-81.

3. Тепловая обработка зерна в установках контактного типа :монография / В.И. Курдюмов, А.А. Павлушин, Г.В. Карпенко, С.А. Сутягин - Ульяновск, УГСХА имени П.А. Столыпина, 2013. -290 c.

4. Математическое моделирование при оптимизации параметров сушильной установки контактного типа / В.И. Курдюмов, А.А. Павлушин, С.Г. Мударисов, В.И. Долгов // Пермский аграрный вестник. - 2016. - № 2(14). - С. 107 - 112.

5. Журавлев, В.А. Оценка эффективности контактной сушки зерна / В.А. Журавлев, А.А. Павлушин // Аграрный потенциал в системе продовольственного обеспечения: теория и практика Материалы Всероссийской научнопрактической конференции. -Ульяновск, УГСХА, 2016. - С. 134-138.

6. К вопросу об определении пропускной способности устройства для сушки зерна / В.И. Долгов, С.А. Сутягин, Г.В. Карпенко, А.А. Павлушин, В.И. Курдюмов // Аграрная наука и образование на современном этапе развития: опыт, проблемы и пути их решения материалы VIII 
международной научно-практической конференции. Ульяновск, 2017. - С. 86-89.

7. Начальные скорости движения частицы материала при перемещении спиральным винтом / Ю.М. Исаев, Х.Х. Губейдуллин, Н.М. Семашкин, И.И. Шигапов // Аграрная наука. -2014. - № 10. - С. 28 - 30.

8. Semashkin, N.M. Revision of the cosmic distance duality tests / N.M. Semashkin, A.V. Nikolaev // 5-ая Ульяновская международная школасеминар “Проблемы теоретической и наблюдательной космологии” / Тезисы докладов. - Улья- новский ГПУ им. И.Н. Ульянова. - 2016. - С. 69.

9. Optimization of research and methodology work at university in terms of the process approach / I.D. Ibragimov, R.R. Iskhakova, M.A. Galeeva, M.M. Kalashnikova, Yu.V. Ryseva, I.I. Galimzyanova, I.A. Sharonov // Journal of Sustainable Development. 2015. - T. 8. - № 3. С. 234-241.

10 Исследование эксцентрикового почвообрабатывающего катка / В.И. Курдюмов, Ю.М. Исаев, И.А. Шаронов, В.Е. Прошкин, А.С. Егоров // Наука в центральной России. - 2016. - № 1 (19). С. 37-45.

\title{
PECULIARITIES OF GRAIN MOVEMENT IN THE DEVICE FOR ITS STIR
}

\author{
Isaev Yu.M., Semashkin N.M., Kalenkov S.A. \\ FSBEI HE Ulyanovsk SAU \\ 432017, Ulyanovsk, Boulevard Novyy Venets, 1; \\ tel .: 8 (84231) 55-95-49, e-mail: emotion.snm@mail.ru
}

\begin{abstract}
Keywords: movement, stirring, throughput capacity, regression equations, speed of grain movement.
The article provides an analysis of theoretical and practical studies of parameters of the device for vertical grain movement in the bulk-grain. As a result, optimal parameters of the device for vertical grain movement and the geometric characteristics of the spiral were obtained. The grain that fills the interturn space of the spiral is in space-limited conditions, as a result, the research on movement of grain material by a spiral-helix working body presents one of the complex experimental and theoretical problems. The theoretical part of the article includes analysis of loose grain flow, for which the expressions of throughput capacity of the stirring device and the speed of grain movement are studied. A spiral screw was installed on the device with the following parameters: outer radius of the spiral screw $r_{2}=35 \mathrm{~mm}$; the pitch of the spiral helix line is $\mathrm{s}=0.07 \mathrm{~m}$; the diameter of the wire from which the spiral was made, $d=0.008 \mathrm{~m}$, the total height of the spiral is $\mathrm{H}=2 \mathrm{~m}$. The device was studied in a wheat heap with a bulk density $\rho=780 \mathrm{~kg} / \mathrm{m}^{3}$. As a result, we obtained an optimal movement speed of the stirring device $u=0,68 \mathrm{~m} / \mathrm{min}$, which is achieved at a spiral frequency of movement of $\omega=930 \mathrm{~min}^{-1}$. Herewith, the throughput capacity of the device is $Q=1,1 \mathrm{~kg} / \mathrm{s}$. The comparison of the experimental and theoretical data obtained showed that the repeatability of the results is $94 \%$. The results of experimental studies prove the validity of the proposed theoretical relatios.
\end{abstract}

\section{Bibliography}

1. Kurdyumov, V.I. Grain disinfection in a plant of combined-type / V.I. Kurdyumov, A.A. Pavlushin, S.A. Sutyagin // Agrarian science as the basis for food security in the region. Materials of the 66th International Scientific and Practical Conference. - Ulyanovsk, USAA, 2015. - P. 181-183.

2. Kurdyumov, V.I. Quality improvement of grain drying in a contact-type plant /V.I. Kurdyumov, A.A. Pavlushin, S.A. Sutyagin // Innovations in Agriculture. -2015. - № 3 (13). - P. 79-81.

3. Heat treatment of grain in devices of contact type: monograph / V.I. Kurdyumov, A.A. Pavlushin, G.V. Karpenko, S.A. Sutyagin. - Ulyanovsk: USAA named after P.A. Stolypin, 2013. - $290 p$.

4.Mathematical modeling of parameter improvement of a drying plant of contact type / V.I. Kurdyumov, A.A. Pavlushin, S.G. Mudarisov, V.I. Dolgov // Perm agrarian vestnik. - 2016. - № 2 (14). - P. 107 - 112.

5. Zhuravlev, V.A. Evaluation of contact grain drying effectiveness / V.A. Zhuravlev, A.A. Pavlushin // Agrarian potential in the food supply system: theory and practice. Materials of the All-Russian Scientific and Practical Conference. - Ulyanovsk, USAA, 2016. - P. 134-138.

6. On the issue of throughput capacity specification of the device for grain drying / V.I. Dolgov, S.A. Sutyagin, G.V. Karpenko, A.A. Pavlushin, V.I. Kurdyumov // Agrarian science and education at the present stage of development: experience, problems and solutions. Materials of the VIII International Scientific and Practical Conference. - Ulyanovsk, 2017. - P. 86-89.

7. Initial velocities of the material particle motion in case of moving with a spiral screw / Yu.M. Isaev, Kh.K. Gubeydullin, N.M. Semashkin, I.I. Shigapov // Agrarian Science. -2014. - № 10. - P. 28 - 30.

8. Semashkin, N.M. Revision of the cosmic distance duality tests / N.M.Semashkin, A.V. Nikolaev //V Ulyanovsk International School-Seminar "Problems of theoretical and observational cosmology": abstracts. - Ulyanovsk STTU named after I.N. Ulyanov .- 2016. - P. 69.

9. Optimization of research and methodology work at university in terms of the process approach / I.D. Ibragimov, R.R. Iskhakova, M.A. Galeeva, M.M. Kalashnikova, Yu.V. Ryseva, I.I. Galimzyanova, I.A. Sharonov // Journal of Sustainable Development. - 2015. - V. 8, No 3. - P. 234-241. 10. Study of an eccentric soil-cultivating roller / V.I. Kurdyumov, Yu.M. Isaev, I.A. Sharonov, V.E. Proshkin, A.S. Egorov // Science in Central Russia. - 2016. - No1 (19). - P. 37-45. 